\title{
Method for Image Portion Retrieval and Display for Comparatively Large Scale of Imagery Data onto Relatively Small Size of Screen Which is Suitable to Block Coding of Image Data Compression
}

\author{
Satellite image (and/or High Definition TV image) retrieval and display for mobile phones
}

\author{
Kohei Arai 1 \\ Graduate School of Science and Engineering \\ Saga University \\ Saga City, Japan
}

\begin{abstract}
Method for image portion retrieval and display for the relatively large scale of imagery data onto comparatively small size of display is proposed. The method is suitable to the data compression methods based on block coding. Through experiments with satellite imagery data, it is found that the proposed method is useful for the display onto small sized screen such as mobile phone display and so on.
\end{abstract}

Keywords-data compression; image retrieval; block coding

\section{INTRODUCTION}

There is a strong demand for displaying remote sensing satellite images onto mobile phone screen. Pixel size of the remote sensing satellite images, in general, is much greater than that of mobile phone screen. The pixel size of mobile phone is less than one million pixels while that of remote sensing satellite images is more than 10 million pixels, in general. On the other hands, the pixel size of High Definition of Television: HDTV ${ }^{1}$ is 1125 by 1080 pixels. Therefore, it is impossible to display HDTV image onto mobile phone screen. Thus scrolling or roaming of the displayed HDTV image or remote sensing satellite image on the mobile phone screen is required. It is obvious that it takes time or a time consumable processes are required to search and display most preferable portion of images on the mobile phone display.

On the other hands, data compression is desirable to reduce the time required for transmission and receiving relatively large size of HDTV images and remote sensing satellite images. One of the image data compression methods is block coding. JPEG ${ }^{2}$ data compression method is one of the well known and widely used block coding methods. JPEG is based on Discrete Cosine Transformation: $\mathrm{DCT}^{3}$ for the block which is composed with 8 by 8 pixels. Namely, images are divided with 8 by 8 pixels of block and DCT is applied to the block by block. DCT allows conversion from image space or time component to frequency component. By eliminating high frequency components, data

\footnotetext{
${ }^{1}$ http://e-words.jp/w/HDTV.html

${ }^{2}$ http://www.jpeg.org/

${ }^{3}$ http://ja.wikipedia.org/wiki/DCT
}

compression can be done. Image portion retrieval and display method proposed here is to search the most preferable portion of images by block by block. Therefore, the proposed search and display method is suitable to block coding of data compression method. The examples of image portion search and display with remote sensing satellite images of Advanced Very High Resolution of Radiometer: AVHRR which onboard NOAA satellites ${ }^{4}$ is demonstrated.

There are a plenty of previous research works on the image retrieval methods. The first microcomputer-based image database retrieval system was developed [1]. Also, survey article documented progresses after 2007 [2]. The authors concentrate efficiency of image retrievals by using geophysical and spatial information of queries [3]-[5]. Also image portion retrieval method is proposed [6].

The following section describes proposed method for image portion retrieval and display method followed by some examples of image portion retrievals and display onto mobile phone screen. Then conclusion with some discussions is described.

\section{PROPOSED METHOD}

\section{A. Process Flow of the Proposed Method}

Process flow of the proposed image portion retrieval and display onto mobile phone screen is shown in Figure 1.

First, the relatively large scale of images such as HDTV, remote sensing satellite images are divided into blocks with 8 by 8 pixels of block. In this process, there two sets of divided blocks of images. The first block of the first divided sub-image starts from the first line/pixel coordinate of the original images while that of the second divided sub-image starts from fourth line/pixel coordinate of the original images as shown in Figure 2.

This image division is replaced and then sub-sub-images, and sub-sub-sub-images are created results in hierarchical

${ }^{4}$ http://noaasis.noaa.gov/NOAASIS/ml/avhrr.html 
structure of the image database as shown in Figure 3. It looks like the well known coarse to fine retrieval method. Thus image portion can be retrieved fast as shown in Figure 4.

Figure 5 shows examples of portion image retrievals with sub-image and sub-sub-image. Namely, image portion retrievals can be done through the layer range from the bottom to the top which is shown in Figure 3.

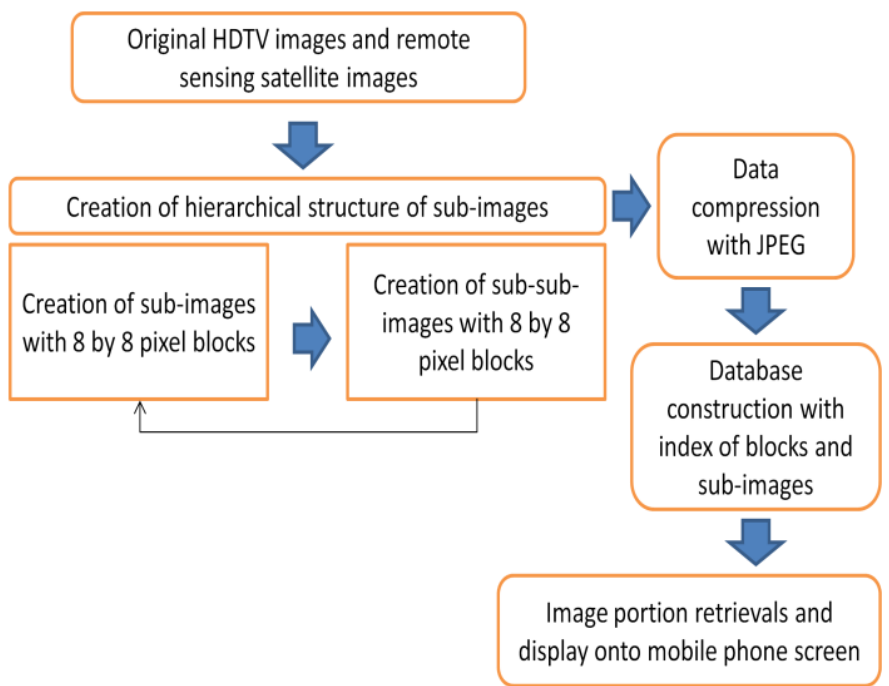

Fig. 1. Process flow of the proposed image portion retrieval and display onto mobile phone screen

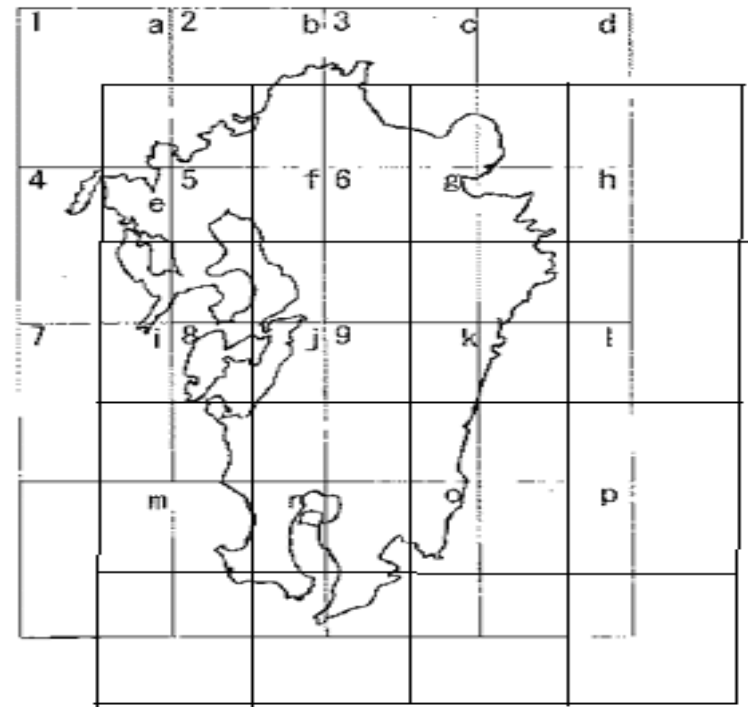

Fig. 2. Original image is divided into two sub-images which are composed with 8 by 8 pixel blocks (the first block of the first sub-image starts from the first line/pixel coordinate while the first block of the second sub-image starts from the fourth line/pixel coordinate)

The specific feature of the proposed image portion retrievals is to use the $50 \%$ overlapped area covers between two sub-image in the same layer. If the area of interest is situated in between two blocks, two adjacent blocks have to be retrieved for the conventional image portion retrievals method while only thing user has to do is to select one of two subimage when they would like to search the areas for the proposed image portion retrieval method.
It makes retrievals based on the proposed method much faster than that for the conventional method.

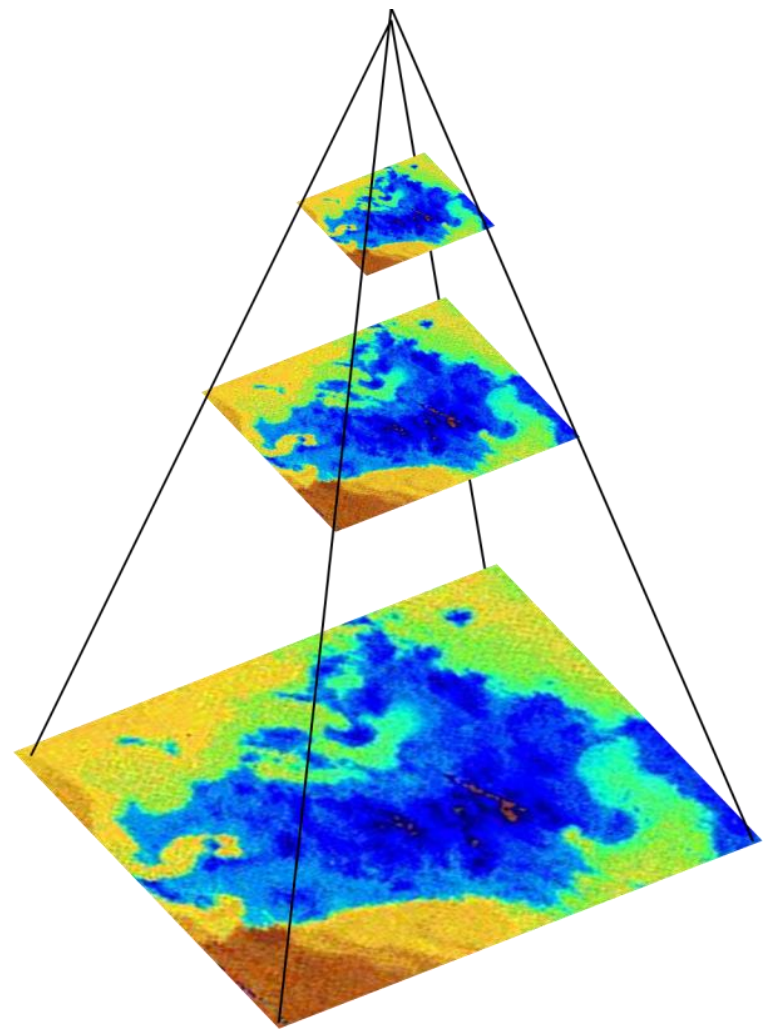

Fig. 3. Hierarchical representation of image database for acceleration of image portion retrievals
(A)

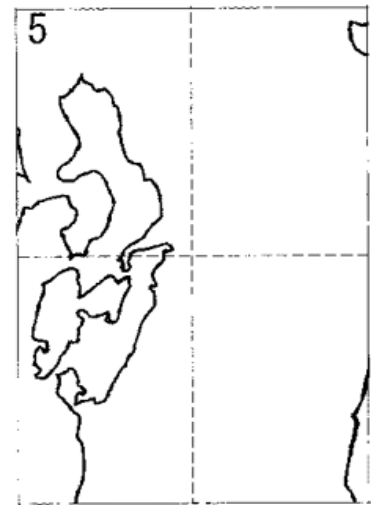

(B)

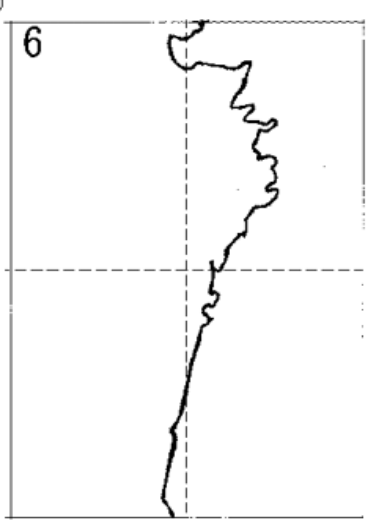

Fig. 4. Examples of sub-sub-images (portion of sub-images)

\section{B. Data Compression and Database}

Second, DCT based JPEG data compression is applied to two sub-images by block by block which composed with 8 by 8 pixels. Then the compressed image data is saved in the image database.

Also, all the blocks in the sub-images are indexed. The blocks in two sub-images are overlapped with $50 \%$. Thus it is possible to retrieve potion of image much faster than the conventional retrieval method with single sub-image. 


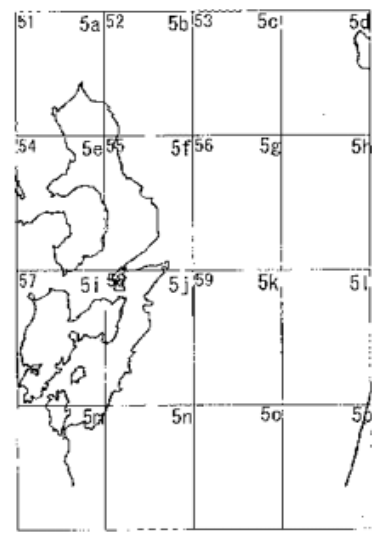

(B)

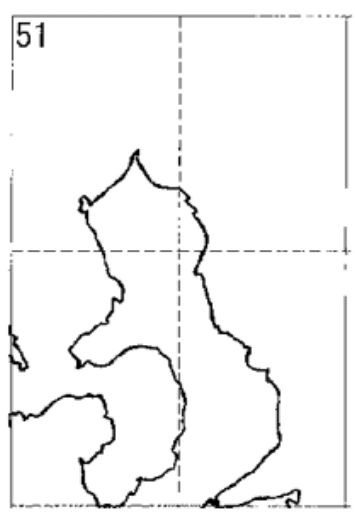

Fig. 5. Examples of portion image retrievals with sub-image and sub-subimage.

\section{EXPERIMENTS}

\section{A. Data Used}

Figure 6 shows the remote sensing satellite images of NOAA/AVHRR derived Sea Surface Temperature: SST which is acquired at $16: 45$ on November 302004 and at 17:11 on May 202002.

\section{B. Portion Image Retrieval and Display onto Mobile Phone \\ Screen}

Figure 7 shows two sub-images, A and B sub-images with the meshes. Users can display low resolution (top layer of image of Figure 3) of whole image onto mobile phone screen at first. Then user goes to search the area of interest through going down to the bottom direction of layer.

After that, users select one of two sub-images depends on their preference. Users can select one of those two sub-image by referencing the area of interest.

Figure 8 (a) shows main menu image of the proposed image portion retrieval system which is displayed onto mobile phone screen while Figure 8 (b) shows retrieved image portion of retrieved result. Users may input retrieval parameters, data product, acquisition time and date.

After that the proposed image portion retrieval system provides the top layered low resolution of whole image. Then users may go down the layer to the bottom layer of a small portion of image in accordance with users' guidance with referencing to the area of interest. When users find out the area of interest is situated in between two adjacent blocks, users have to switch the sub-image from A to B, or B to A.

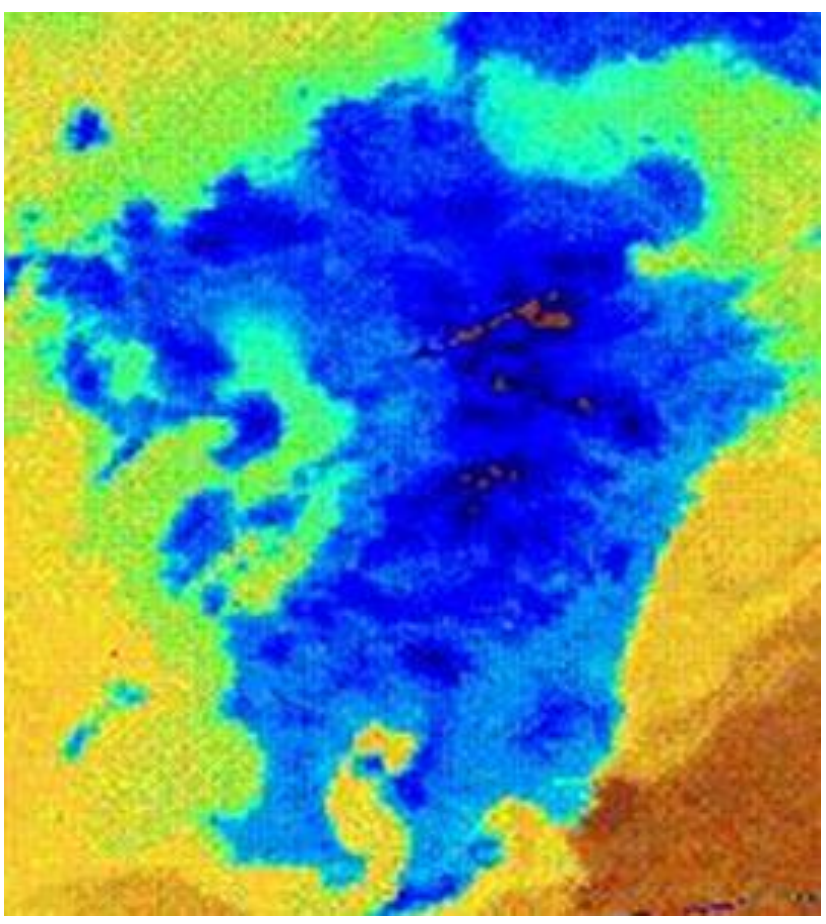

(a) SST at 16:45 on November 302004

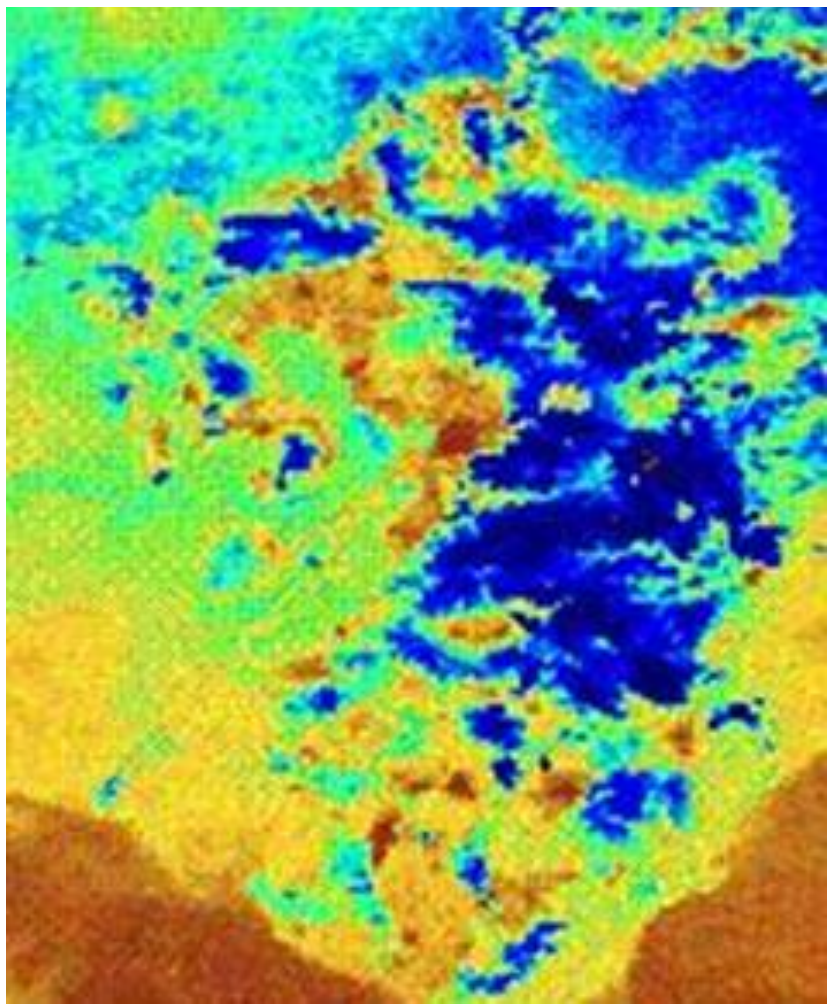

(b) SST at 17:11 JST on May 202002

Fig. 6. Remote sensing satellite images used for experiments 


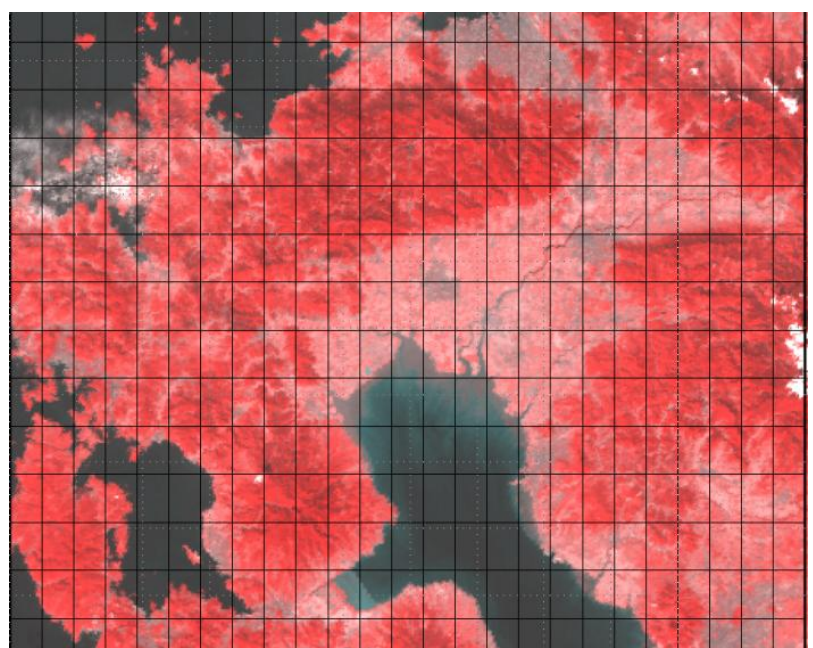

(a)A sub-image

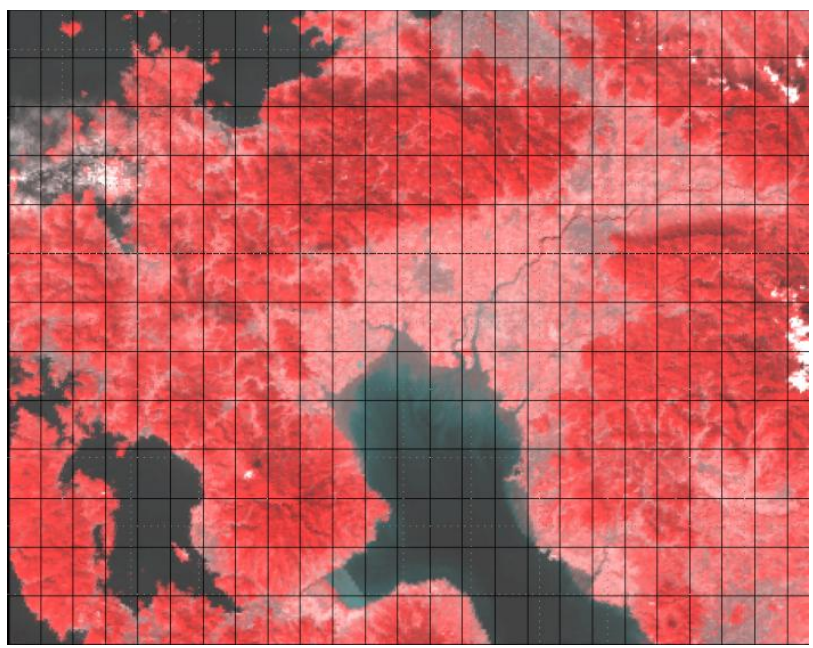

(b)B sub-image

Fig. 7. shows two sub-images with the meshes.

\section{CONCLUSION}

Method for image portion retrieval and display for the relatively large scale of imagery data onto comparatively small size of display is proposed. The method is suitable to the data compression methods based on block coding. Through experiments with satellite imagery data, it is found that the proposed method is useful for the display onto small sized screen such as mobile phone display and so on

It is found that the proposed image portion retrieval and display method and system works well in terms of the time required for retrievals. This is caused by using $50 \%$ overlapping two sub-images.

\section{ACKNOWLEDGMENT}

The author would like to thank Mr. Kazuo Kojima for his effort to conduct image portion retrieval experiments.

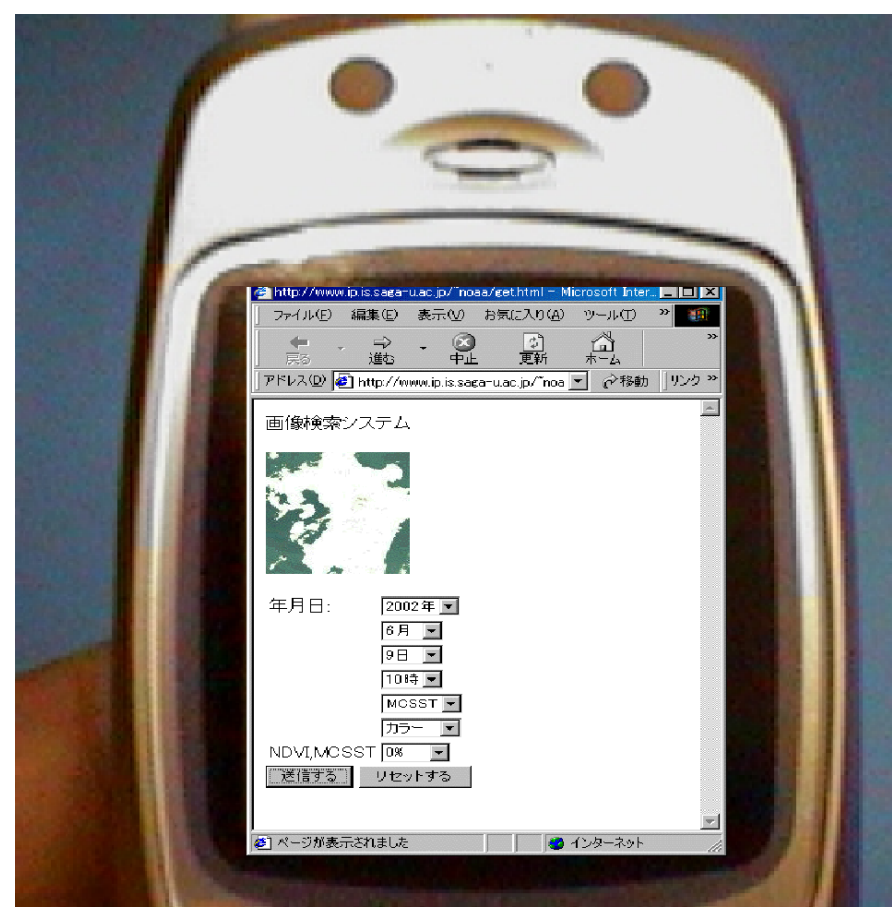

(a)Main menu of the proposed image portion retrieval system

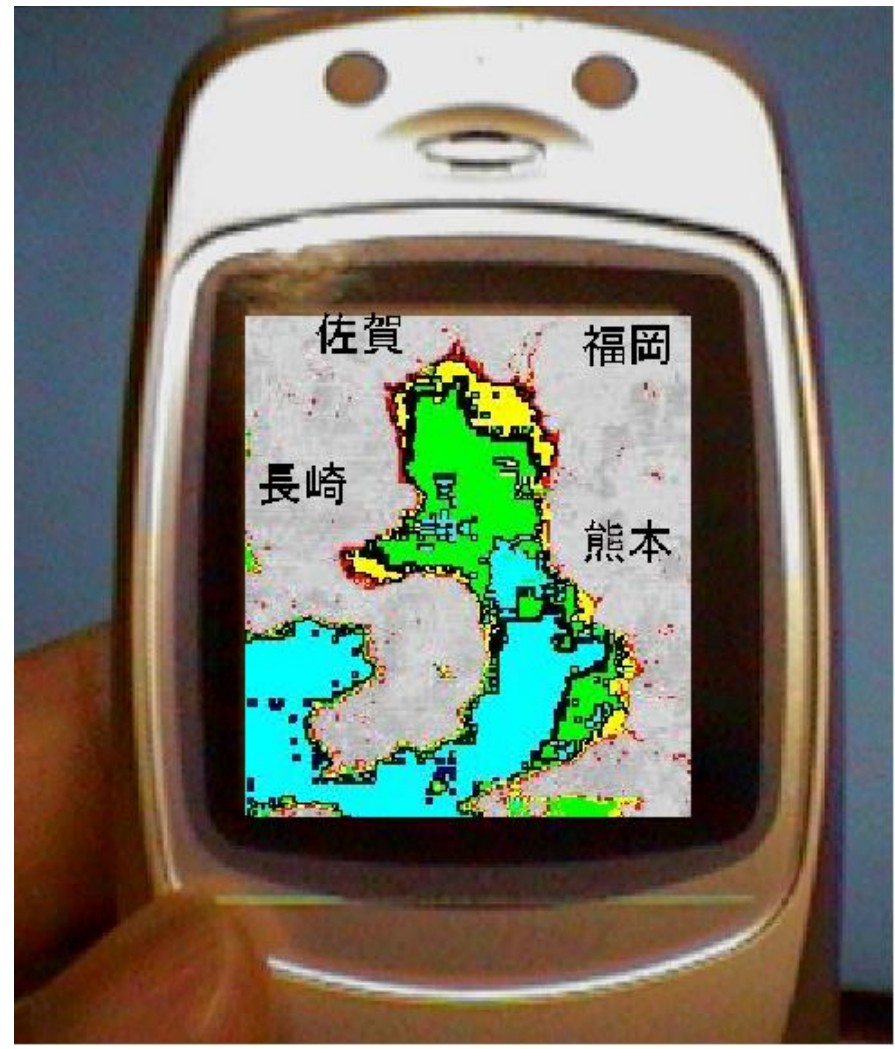

(b)Displayed SST image as retrieved result

Fig. 8. Displayed image onto mobile phone screen. 


\section{REFERENCES}

[1] Prasad, B E; A Gupta, H-M Toong, S.E. Madnick, A microcomputerbased image database management system, IEEE Transactions on Industrial Electronics IE-34, 1, 83-8, 1987.

[2] Datta, Ritendra; Dhiraj Joshi, Jia Li, James Z. Wang, Image Retrieval: Ideas, Influences, and Trends of the New Age, ACM Computing Surveys 40, 2, 1-60, 2008.

[3] K. Arai, H. Eto, T. Nishiyama, Remote sensing satellite image database system which alolows retrievals with fuzzy queries, Journal of Japan Society of Photogrammetry and Remote Sensing, 38, 4, 47-52, 1999.

[4] K. Arai, M. Arakawa, H. Eto, Fuzzy retrievals of remote sensing satellite image database based on fuzzy theory utilizing geophysical and spatial information related queries, Journal of Japan Society of Photogrammetry and Remote Sensing, 38, 4, 17-25, 1999.

[5] H. Eto, T.Yamamoto, K.Arai, Image indexing for image retrievals utilizing spatial information of queries, Journal of Japan Society of Photogrammetry and Remote Sensing, 39, 3, 14-20, 2000.
[6] K. Arai, Image portion retrieval and display large size of images onto small size of display, Report from the Science and Engineering Faculty, Saga University, 32, 2, 7-14, 2007.

\section{AUTHORS PROFILE}

Kohei Arai, He received BS, MS and PhD degrees in 1972, 1974 and 1982, respectively. He was with The Institute for Industrial Science, and Technology of the University of Tokyo from 1974 to 1978 also was with National Space Development Agency of Japan (current JAXA) from 1979 to 1990. During from 1985 to 1987 , he was with Canada Centre for Remote Sensing as a Post Doctoral Fellow of National Science and Engineering Research Council of Canada. He was appointed professor at Department of Information Science, Saga University in 1990. He was appointed councilor for the Aeronautics and Space related to the Technology Committee of the Ministry of Science and Technology during from 1998 to 2000. He was also appointed councilor of Saga University from 2002 and 2003 followed by an executive councilor of the Remote Sensing Society of Japan for 2003 to 2005. $\mathrm{He}$ is an adjunct professor of University of Arizona, USA since 1998. He also was appointed vice chairman of the Commission " $\mathrm{A}$ " of ICSU/COSPAR in 2008. He wrote 30 books and published 332 journal papers. 